\title{
Oncogenic Osteomalacia misdiagnosed as Ankylosing Spondylitis
}

Tanrıkulu S., Selçukbiricik Ö., İnce B., Üzüm A.K., Gül N., Tekin S., Aral F., Tanakol R

Istanbul University, Istanbul Faculty of Medical, department of Internal Medicinei division of Endocrinology and Metabolism

\section{Introduction:}

Oncogenic osteomalacia is a rare paraneoplastic syndrome, which can be associated with phosphaturic mesenchymal or non-mesenchymal tumors. These tumors produce fibroblast growth factor 23 (FGF-23) that leads to subsequent hypophosphatemic osteomalacia. We, hereby present a patient with oncogenic osteomalasia who is misdiagnosed as ankylosing spondylitis.

\section{Case:}

A 54-year old man with weakness, severe arthralgia involving pelvis, hip joints and lower extremities referred to the Rheumatology Department for the treatment of a suspected sero-negative spondiloarthritis and TNF-alpha inhibitor and glucocorticoid treatment had begun. Despite of three years of treatment, there was no relief of symptoms, and laboratory tests had shown hypophosphatemia $(0,7 \mathrm{mg} / \mathrm{dl})$ with low vitamin D and normal calcium, PTH levels, referred to Endocrinology clinic. Hypophoshatemia was resistant to oral phospate replacement. Physical examination revealed a non-tender, mobile, $2 \mathrm{~cm}$ nodular lesion at right medial thigh. Magnetic resonance imaging showed an ovular $2.1 \times 1.7 \mathrm{~cm}$ hypointense solid lesion with irregular borders in the soft tissue at right thigh. FDG-PET scanning showed high FDG uptake at the lesion, and no other hypermetabolic masses.

We performed tumor resection and after an unremarkable post-operative course hypophospatemia resolved within 2 weeks. Histopathologic evaluation showed extraosseos giant cell tumor with metaplastic bone cell formation. After surgical removal patient's symptoms have revealed and phosphorous levels became to normal levels.

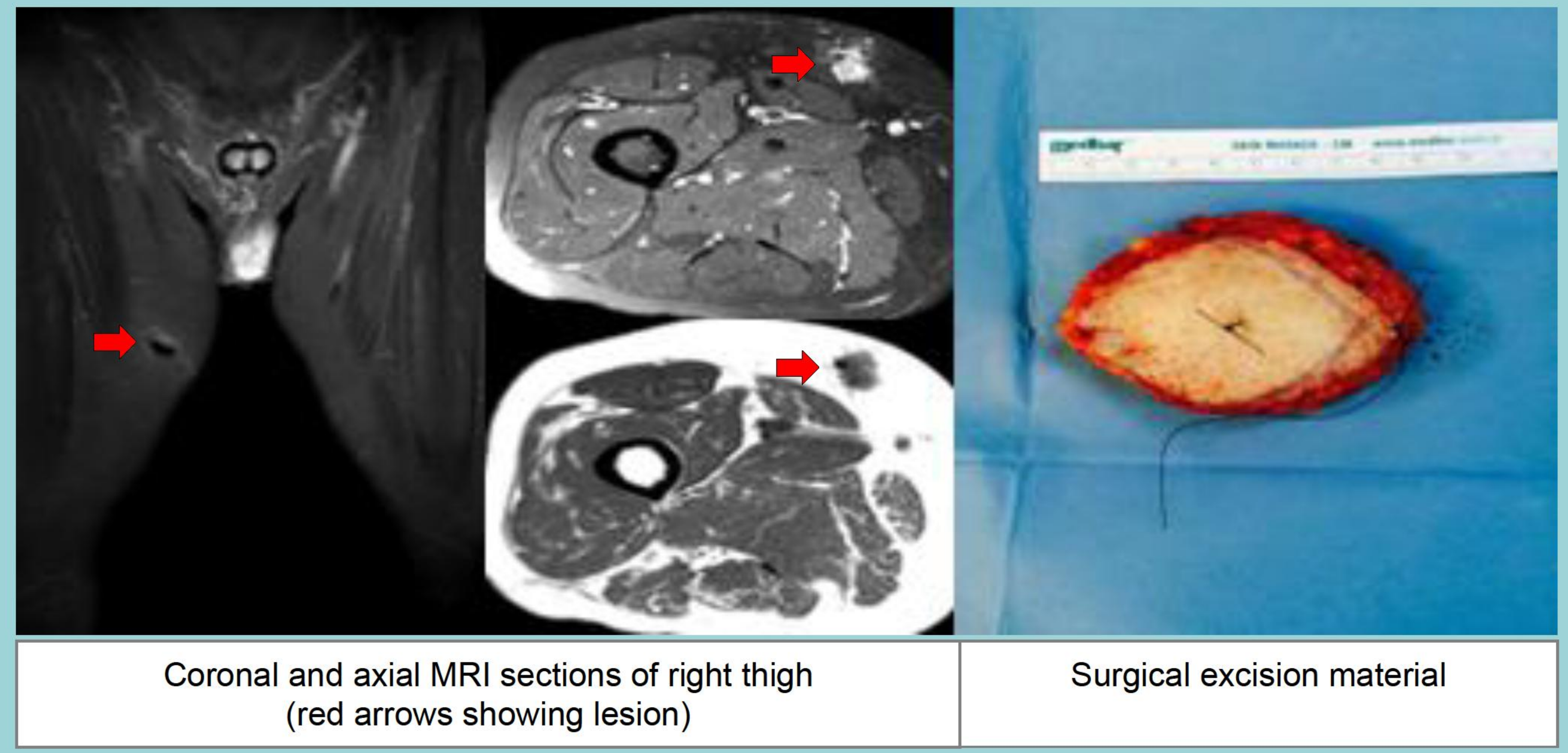

Conclusions:

Oncogenic osteomalacia and ankylosing spondylitis, both can present with arthralgia, weakness, difficulty in walking. For the patients who have features suggestive of oncogenic osteomalacia, it is of crucial importance to perform a survey to detect the tumour or tumours responsible.

\section{References:}

1- Chong $\mathrm{WH}$, Tumor localization and biochemical response to cure in tumor-induced osteomalacia. J Bone Miner Res. 2013 Jun;28(6):1386-98. 